\title{
FUNCIONES Y SITUACIÓN ACTUAL DE LA INTERVENCIÓN DE LOS PSICÓLOGOS EN CUIDADOS PALIATIVOS
}

\section{FUNCTIONS AND CURRENT SITUATION OF THE INTERVENTION OF PSYCHOLOGIST IN PALLIATIVE CARE}

\author{
Manuel Fernández-Alcántara' ${ }^{1}$, Azucena Ortega-Valdivieso'ㄹ, M. Nieves Pérez-Marfil', $M^{a}$ \\ Paz García-Caro ${ }^{3}$ y Francisco Cruz-Quintana \\ ${ }^{1}$ Departamento de Personalidad, Evaluación y Tratamiento Psicológico. Facultad de Psicología. Universidad \\ de Granada \\ 2 Departamento de Fisioterapia. Facultad de Ciencias de la Salud. Universidad de Granada \\ ${ }^{3}$ Departamento de Enfermería. Facultad de Ciencias de la Salud. Universidad de Granada
}

\section{Resumen}

Los psicólogos tienen un papel crucial en los procesos de fin de vida. Dentro de éstos, los cuidados paliativos son una de las áreas con más desarrollo en los últimos años, proponiendo un cuidado holístico e integral. Sin embargo, las funciones y roles de los psicólogos no están del todo claros. Por ello, se ha realizado una revisión teórica, buscando los estudios que ponen de relevancia su papel, funciones, así como la formación específica que deberían tener los psicólogos que trabajen en esta área. La mayoría subrayan la triple intervención con el paciente, la familia y el equipo sanitario. Funciones de evaluación e intervención psicológica, así como de prevención del burnout y del duelo aparecen como destacadas. La formación no está reglada aunque se está intentando buscar un currículum común.

Palabras clave: Psicólogo, cuidados paliativos, psicología, psicoterapia, fin de vida.

\section{Abstract}

Psychologists have a crucial role in endof-life processes. Within them, palliative care is one of the most developed areas and it is based on an holistic approach. However, the role of psychologists in this area is not clear. In order to clarify their function, a theoretical review has been carried out. The objective was to identify and integrate into a narrative the roles and functions of psychologist working in palliative care, as well as topics related to specific formation. Most of the articles reviewed outlined an intervention based on three axis: the patient, the family and the health care team. Functions such as psychological assessment, prevention of burnout and intervention on bereaved people appears as central. Specialized training and formation is not regulated, although recent proposals are trying to elaborate a common curriculum.

Keywords: Psychologist, palliative care, psychology, psychotherapy, end of life care.

\section{Correspondencia:}




\section{PLANTEAMIENTO DEL PROBLEMA}

Los Cuidados Paliativos (CP) se definen como una asistencia dirigida al creciente número de personas con enfermedades crónico-degenerativas y con cáncer que padezcan al final de su vida un sufrimiento intenso y precisen de atención sanitaria y social especial (1). Este tipo de cuidados no tiene por objetivo alargar la vida a la persona, sino conseguir una calidad de vida lo más alta posible, que pueda permitir tener lo que se denomina "buena muerte" o "muerte digna"(2,3). Así, se señala como principal objetivo de los CP el bienestar de la persona, y no la curación de la enfermedad ${ }^{(1,4-7)}$.

Una de las líneas estratégicas en el Plan de Cuidados Paliativos propuesta desde el Sistema Nacional de Salud ${ }^{(1,7)}$, es la atención integral tanto a pacientes como a familiares en procesos de final de vida. Aquí se especifica la necesidad de un abordaje holístico, donde tengan cabida el control de síntomas, los aspectos emocionales, sociales y espirituales; la prevención del duelo; la promoción del trabajo multidisciplinar así como la coordinación intra e inter niveles dentro del sistema asistencial; o el fomento de la comunicación entre los implicados ${ }^{(8,9)}$.

Para dar respuesta a las diferentes necesidades del paciente, es necesaria una estrategia de carácter multidisciplinar, donde cada profesional de la salud pueda asesorar y acompañar de la manera más óptima. Por ello, se plantea que habrá funciones y tareas que serán desempeñadas por todo el equipo multidisciplinar, mientras que otras requerirán una formación específica ${ }^{(10,11)}$.

Las situaciones que se plantean en el fin de vida están caracterizadas por su dificultad y su complejidad, y por generar momentos de gran desestructuración emocional a los que hay que dar respuesta $y$, para los que no todos los profesionales están formados y preparados ${ }^{(8)}$. Los psicólo- gos, en este sentido, pueden aportar tanto herramientas propias como intervenciones específicas. Ejemplos de ellas son: el acompañamiento en los procesos de duelo ${ }^{(12)}$, la evaluación psicológica ${ }^{(13-16)}$, el trabajo con la subjetividad de la persona ${ }^{(11,17,18)}$, sin olvidar las problemáticas de las familias y el

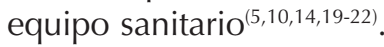

Respecto a la composición profesional de los equipos de CP en España, se informa que aproximadamente la mitad disponen de un psicólogo ${ }^{(9,23)}$. Esta cifra ha estado aumentando desde $1998^{(8)}$, situando a España como el segundo país en Europa con más psicólogos trabajando en $\mathrm{CP}^{(20)}$.

Las funciones que debería realizar el psicólogo en las unidades de CP, al igual que los modelos utilizados, varían mucho dependiendo de cada autor. Mientras que en algunos casos se utilizan modelos centrados en el carácter temporal| ${ }^{(13)}$, otros se organizan por niveles de intervención ${ }^{(11,20,21)}$, o por tareas $^{(24)}$. Lo mismo ocurre con la formación, ya que aunque empiezan a aparecer trabajos donde se plantea la necesidad de crear un currículum común ${ }^{(20,25,26)}$, lo que se observa es una falta de acuerdo en los contenidos, fruto de la diversidad de funciones y aproximaciones.

No se ha encontrado ningún documento que refleje de manera integrada los diferentes roles y funciones del psicólogo. Por ello, el objetivo de este estudio es revisar las publicaciones científicas realizadas en los últimos años en relación a la situación, la formación, y funciones del psicólogo en el área de los CP.

Para cumplir dicho objetivo se realizó una búsqueda sobre artículos científicos publicados en las bases de datos PubMed, PsycINFO, CINAHL, Scopus y Web of Science. Se ha decidido indicar primero el proceso general empleado, e ir descendiendo en el nivel de profundización explicativa. Las estrategias de búsqueda para identificar la producción científica fueron diferentes, teniendo en cuenta si la base de datos po- 
see o no lenguaje controlado Thesauro. Tres de las bases de datos poseen lenguaje normalizado: PubMed, Psyclnfo y CINAHL. En la tabla 1 se presentan los descriptores de cada tesauro para los términos psychologist y palliative care. No se optó por añadir una tercera categoría que incluyera términos relacionados con roles y/o funciones debido a la excesiva especificidad y a los escasos resultados obtenidos.

Dos bases de datos no poseen lenguaje normalizado: Scopus y Web of Science. Para elaborar las ecuaciones de búsqueda nos servimos de los descriptores Thesauro (tabla 2).

Así mismo, en Scopus se optó por limitar las búsquedas dentro del título y las palabras clave (TITLE-KEY), eliminando la opción abstract (ABS), puesto que incluía demasiado ruido documental. Tras estudiar las diferentes posibilidades de consulta en estas dos bases de datos, las ecuaciones de búsqueda elegidas fueron las que se muestran en la tabla 3.

Sobre los resultados obtenidos de cada base de datos se realizó una revisión manual, para determinar cuáles de ellos estaban directamente relacionados con el tema de estudio. Posteriormente, tras la obtención de los artículos se revisaron las referencias bibliográficas de éstos para obtener otra serie de artículos relacionados con la temática, que no se encontrasen en las bases de datos señaladas anteriormente.

\section{Tabla 1. Descriptores utilizados}

\begin{tabular}{lll}
\hline Base de datos & Descriptor "Psychologist" & Descriptor "Palliative Care" \\
\hline PubMed & "Psychology" & "palliative care" \\
& "Psychologists" & "Palliative treatment" \\
& "Psychologist" & "palliative therapy" \\
& "Psychological Therapy" & "Palliative" \\
\hline Psyclnfo & "Psychologist" & "Terminally" \\
& "Psychologists" & "Palliative Care" \\
\hline CINAHL & "Mental Health Professional" & "Hospice Care" \\
& & "Terminal Care" \\
& "Hospice and Palliative Nursing" \\
\hline
\end{tabular}

Tabla 2. Términos de búsqueda

\begin{tabular}{cc}
\hline Base de datos & Términos de búsqueda \\
\hline "Psycholog*" \\
"Palliative Care" " \\
"Terminally ill" \\
"Terminal illness" \\
"end of life" \\
"hospice" \\
"palliative therapy" \\
\hline
\end{tabular}


Tabla 3. Ecuaciones de búsqueda para cada una de las bases de datos

\begin{tabular}{|c|c|}
\hline Base de Datos & Ecuaciones utilizadas \\
\hline PubMed & $\begin{array}{l}\text { ("palliative therapy" [MeSH Terms]) } \\
\text { ("palliative" [MeSH Terms] AND ("psychological therapy"[MeSH Terms]) } \\
\text { ("palliative therapy" [MeSH Terms] AND ("psychology" [MeSH Terms]) } \\
\text { ("palliative care" [MeSH Terms] AND ("psychology"[MeSH Terms]) } \\
\text { (psychologist[MeSH Terms]) OR (psychologists[MeSH Terms]) AND (palliative } \\
\text { care[MeSH Terms]) OR palliative treatment[MeSH Terms]) OR (palliative } \\
\text { therapy[MeSH Terms]) OR (palliative medicine[MeSH Terms]) }\end{array}$ \\
\hline Psyclnfo & $\begin{array}{l}\text { (palliative and psychologists).HW } \\
\text { (terminally and psychologists).HW }\end{array}$ \\
\hline CINAHL & $\begin{array}{l}\text { (MM "Palliative Care") AND (MM "Hospice and Palliative Nursing") AND } \\
\text { (MM "Psychologists") } \\
(\mathrm{MM} \text { "Palliative Care") AND (MM "Psychologist") }\end{array}$ \\
\hline Scopus & $\begin{array}{l}\text { (TITLE(psycholog*) AND DOCTYPE (ar OR re)) AND ((TITLE- ABS- } \\
\text { KEY("palliative care") AND DOCTYPE (ar OR re)) OR (TITLE- ABS-KEY } \\
\text { ("terminally ill") AND DOCTYPE (ar OR re)) OR (TITLE- ABS-KEY ("terminal } \\
\text { illness") AND DOCTYPE (ar OR re)) OR (TITLE- ABS-KEY ("end of life") AND } \\
\text { DOCTYPE (ar OR re)) OR (TITLE- ABS-KEY ("hospice") AND DOCTYPE (ar } \\
\text { OR re)) OR (TITLE- ABS-KEY ("palliative therapy") AND DOCTYPE (ar OR re))) }\end{array}$ \\
\hline Web of Sciene & 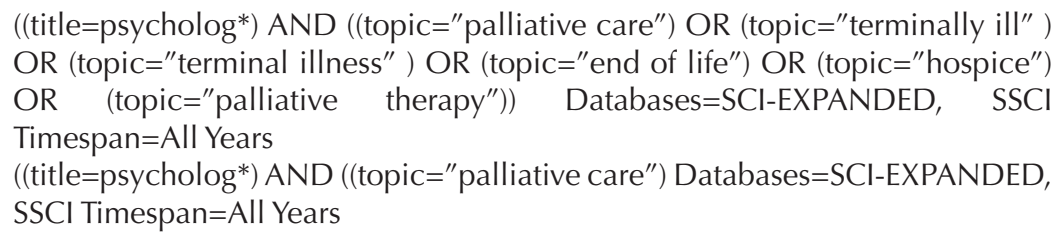 \\
\hline
\end{tabular}

La búsqueda bibliográfica fue realizada entre los meses de enero de 2012 y septiembre de 2012, posteriormente se realizó una actualización en Julio de 2013. Los artículos se seleccionaron siguiendo los siguientes criterios de inclusión:

- Artículos publicados en inglés, español o francés en revistas científicas. Debido al escaso número de artículos encontrados no se realizó una selección por año de publicación.

- El objetivo de este estudio fue conocer en profundidad el papel de los psicólogos que trabajan en cuidados paliativos. Por ello, no se han tenido en cuenta aquellos que estudian las funciones e intervenciones de otro tipo de personal sanitario (médicos y enfermeros principalmente), ni los que versan sobre las necesidades de los pacientes que reciben $\mathrm{CP}$.

\section{DESARROLLO DEL TEMA}

\section{Situación de los psicólogos en CP}

Actualmente el psicólogo se encuentra preso de dos discursos diferentes, en lo que se refiere a su trabajo en los procesos de fin de vida: por un lado se habla de su importancia y se ve como un elemento básico y necesario en cualquier intervención, mientras que por otro se señala que sus 
funciones pueden ser realizadas por otros miembros del equipo ${ }^{(10,20)}$. Esta situación aparece relacionada con el escaso número de psicólogos que trabajan en este área y las dificultades asistenciales, que se presentan, para llevar a cabo su intervención ${ }^{(27-29)}$. El estudio de Nydegger, pone en evidencia esta cuestión, no encontrando diferencias significativas con respecto al número de psicólogos trabajando en hospices, comparada con la medida que realizó el mismo autor diez años antes ${ }^{(30)}$.

Estudios realizados por psicólogos clínicos, muestran que el porcentaje de pacientes remitidos para algún tipo de intervención psicológica era de aproximadamente el $11 \%{ }^{(31)}$. Este dato puede ser aún más sorprendente si se compara con otro estudio realizado en España, en el que unos jueces tenían que evaluar el grado de desesperación en que se hacía la demanda de intervención ${ }^{(32)}$. Los resultados destacaron que un $31,7 \%$ de las demandas analizadas, habían sido catalogadas como desesperadas o bastante desesperadas. Esto, unido al poco tiempo de que se dispone para estar con el paciente, dificulta aún más la tarea del psicólogo ${ }^{(32)}$.

Los psicólogos también han señalado la existencia de diferentes obstáculos durante su intervención en el final de vida. Las propias emociones, los procesos de identificación o la organización del sistema sanitario pueden ser causa de dificultades durante el apoyo y acompañamiento emocional. Además, el ambiente en la unidad de trabajo es señalado como un importante determinante de los niveles de satisfacción en los psicólogos que trabajan en cuidados paliativos ${ }^{(29)}$.

\section{Modelos explicativos de las funciones e intervenciones del psicólogo}

Las fuentes bibliográficas consultadas sugieren, por un lado, la existencia de modelos teóricos que pretenden explicar las funciones del psicólogo, y por otro, estudios descriptivos donde se les pide a los psicólogos que indiquen cuál es su trabajo dentro de estas unidades.

Con respecto a los modelos, uno de los pioneros fue el enunciado por Haley et al. ${ }^{(13)}$. Los autores proponen una visión temporal de la intervención del psicólogo, diferenciando cuatro momentos: antes de que aparezca la enfermedad terminal (labores de prevención y educativas, centradas en la toma de conciencia de la muerte), durante el diagnóstico (intervenciones centradas en la problemática psicológica/ existencial del paciente, así como labores de formación con el equipo), cuando la enfermedad ya se encuentra en estado avanzado (detectando problemas en la familia, continuando con la intervención psicológica) y finalmente durante el periodo posterior a la pérdida (situaciones inconclusas, así como duelos complicados o problemas en el equipo sanitario).

Otro modelo surge del intento por definir un currículum común para los psicólogos en $\mathrm{CP}^{(20)}$. Dicha aproximación se basa en una estructura piramidal de cuatro niveles donde se especifican las labores de los diferentes profesionales sanitarios que atienden en el fin de vida. Según estos autores, el psicólogo estaría centrado en los niveles III y IV, que corresponden a las intervenciones específicas de counselling, manejo de la ansiedad, o a técnicas más específicas (como la psicoterapia propiamente dicha). Los niveles restantes (I y II) se caracterizarían por el uso de técnicas generales de apoyo emocional, algo que puede ser realizado por el psicólogo, y por el resto de personal que trabaje en CP. Desde este planteamiento, el equipo multidisciplinar debería de disponer de los suficientes recursos y habilidades para realizar un acompañamiento y un soporte emocional, así como para detectar los casos de mayor complejidad emocional, que serían susceptibles de una evaluación e in- 
tervención más específica por parte de los psicólogos que trabajen en la unidad ${ }^{(33)}$.

Este modelo guarda cierto parecido con aquel que proporcionan Payne y Haines ${ }^{(14)}$. Las autoras hablan sobre tres niveles de cuidado psicológico, cada uno de los cuales necesitaría de unas destrezas específicas para llevarse a cabo. El primero de ellos supone la utilización de habilidades básicas de comunicación y relación (mediante el uso de técnicas intuitivas, así como de counselling más complejas, siendo necesaria una formación específica. Por último, el tercer nivel requiere un amplio conocimiento teórico/práctico de multitud de teorías, y se caracteriza por poder adaptar las habilidades a la problemática del paciente.

Finalmente, el modelo propuesto por Bayés en diferentes artículos subraya la existencia de otros tres niveles ${ }^{(2,5,11)}$. El primero sería el de detección del problema e incluiría las habilidades básicas que ya hemos comentado, el segundo nivel se centraría en la valoración de los síntomas psicológicos refractarios, tratamiento de la angustia o del duelo. Por último, el tercer nivel de intervención comprendería a un psicólogo exterior al servicio de CP que realizaría intervenciones en la dinámica del equipo.

\section{Funciones del psicólogo en una unidad de Cuidados Paliativos}

Los modelos analizados son interesantes propuestas que pretenden recoger parte de las actividades del psicólogo en los CP. Por otra parte, diversos autores han señalado muchas de las funciones del psicólogo, sin establecer modelos ${ }^{(21,30,31)}$. En este sentido, hay que destacar el importante trabajo realizado por Lacasta et al. ${ }^{(9)}$. Estos autores, enviaron una encuesta a 262 equipos de cuidados paliativos, para indagar sobre cómo se realizaban las distintas labores del psicólogo. El estudio es una réplica del realizado por la misma autora unos años antes $^{(34)}$. Los resultados actuales muestran que la principal función que realizan, es la atención psicológica, tanto al paciente como a su familia. Por detrás de ésta se encuentran el asesoramiento emocional al personal e intervenciones en caso de burnout. Labores de coordinación, así como de docencia e investigación son otras de las funciones más relevantes. También se observa como el porcentaje de pacientes sobre los que intervienen se ha modificado, siendo los oncológicos el 50\%, y constituyendo el otro $50 \%$ pacientes con diferentes diagnósticos como enfermedad crónica, neurológica, o geriátrica, todas de carácter degenerativo.

Se encuentra un importante volumen de trabajos, realizados en Francia, sobre las funciones del psicólogo dentro del equipo multidisciplinar. La mayoría de estas investigaciones están orientadas desde una aproximación psicodinámica, y ponen de relevancia aspectos relacionados con la subjetividad $^{(35)}$. Se destaca la función del psicólogo como un tercero, como el encargado de subrayar el deseo y articular la demanda del paciente ${ }^{(18,36-39)}$. Así mismo, señalan que la práctica del psicólogo debería de ser repensada de manera continua, y estar basada en la escucha e identificación de procesos psíquicos de carácter inconsciente ${ }^{(40,41)}$. El psicólogo actuaría como un facilitador, ante las posibles dificultades del paciente al enfrentarse a la inminencia de la muerte ${ }^{(42,43)}$. Finalmente, nos encontramos con trabajos en los que se reflexiona sobre el uso de ciertas técnicas específicas por parte de los psicólogos, como pueden ser los escritos sobre sus impresiones con los pacientes en proceso de final de $v^{2} a^{(44)}$, o sobre la necesidad de colaboración en equipos multidisciplinares donde el reconocimiento mutuo es necesario ${ }^{(45)}$.

Las intervenciones del psicólogo en los aspectos emocionales tanto del paciente 
como de sus familiares en el final de la vida son valoradas muy positivamente tanto por los propios pacientes como por otros profesionales sanitarios. Ibáñez del Prado et al. ${ }^{(46)}$ señalaron que prácticamente la totalidad de los enfermos (98\%) y de los cuidadores (100\%) recibieron un apoyo emocional adecuado a través de la intervención psicológica, obteniendo beneficios de la misma. Duro-Martínez et al. ${ }^{(47)}$ destacaron el importante papel de la asistencia psicológica en cuidados paliativos, especialmente en aquellos casos más complejos: cuando niños o adolescentes están involucrados, ante dinámicas familiares que favorecen la conspiración de silencio, o en procesos de duelo complicado ${ }^{(47,48)}$.

Finalmente, parece importante señalar las necesidades del propio psicólogo. Hay estudios que indican la utilidad de realizar una revisión personal sobre el tema de la muerte y cómo la concibe ${ }^{(17,24)}$. Así mismo, son los propios psicólogos los que señalan la necesidad de trabajar estos aspectos y de disponer de grupos de palabra que les ayuden a poder sostenerse en el acompañamiento de estos pacientes ${ }^{(29)}$.

\section{Implicaciones formativas para el psicólogo en los procesos de fin de vida}

La formación se sitúa como una temática básica y emergente en el final de la vida ${ }^{(20)}$. En la actualidad no existe una formación reglada, por lo que nos encontramos con multitud de propuestas, que difieren bastante entre $\mathbf{s i ́}^{\prime 25,26)}$.

Hay muchos factores que habría que tener en cuenta para poder establecer un consenso, entre los que destaca el gran elenco de patologías a las que tiene que hacer frente el psicólogo y que van más allá de la enfermedad oncológica. Así mismo, sería necesario entrenar un gran número de habilidades y herramientas, asociadas a la realización de las diversas funciones del psicólogo en este área ${ }^{(10,49)}$. Como bien señalan Jünger et al. ${ }^{(20)}$ habría que establecer claramente los roles del psicólogo con respecto a los pacientes, la familia y el personal. Así mismo, es necesario tener en cuenta las diferencias culturales, a la hora de establecer la posibilidad de un currículum común. Una de las tareas pendientes es la actualización de los trabajos referidos a la formación en CP en España ${ }^{(25,26)}$.

\section{DISCUSIÓN / CONCLUSIONES}

La intervención psicológica en cuidados paliativos debe de estar basada en un modelo de atención que tenga en cuenta las necesidades biológicas, psicológicas, sociales y existenciales. Aunque cada persona dentro del equipo va a ser especialista en una o varias de ellas, ninguno de los miembros debería perder de vista esta aproximación holística.

La intervención del psicólogo está dirigida a tres ejes principales: el paciente, la familia y el equipo profesional; y estando presente a lo largo de una línea temporal (13), que va desde las labores preventivas hasta el trabajo que se produce con las familias tras el fallecimiento del paciente. Existen intervenciones emocionales compartidas por el personal sanitario que interviene en el fin de vida, así como otras de carácter específico, conformando una pirámide, de forma análoga a los diferentes niveles de intervención propuestos por ciertos autores ${ }^{(14,20)}$.

Es necesario que se tengan en cuenta los factores subjetivos que influyen en el acompañamiento emocional, así como las diversas necesidades y obstáculos que pueden tener los psicólogos al trabajar en cuidados paliativos ${ }^{(29)}$. Algunas soluciones posibles son la supervisión de la práctica, así como la conciencia de las propias actitudes y creencias con respecto a la muerte, debido a la influencia que puede tener en el proceso de acompañar a estos pacientes. 
La principal limitación de este estudio de revisión han sido las bases de datos consultadas, ya que cabe la posibilidad de que ciertos artículos que se encuentren en otros buscadores no estén recogidos.

Con respecto a las nuevas líneas de investigación, sería necesario realizar estudios de corte cualitativo que permitan ver cuáles son los obstáculos y problemáticas que encuentran los psicólogos en su práctica y trato con el sufrimiento y la muerte (50,51). Es decir, pasar de la dimensión de qué es lo que hacen, a preguntarnos cómo lo hacen, cuáles son sus experiencias y sus emociones al trabajar en CP. Así mismo, sería necesario estudiar cómo se organiza el equipo multidisciplinar, y cuáles son las áreas donde necesitan una mayor intervención psicológica. Un ejemplo de esto sería la investigación que siguen autores como Serra ${ }^{(19)}$ y Ortega et al. ${ }^{(22)}$ sobre intervenciones psicológicas para disminuir los niveles de burnout en los CP.

\section{REFERENCIAS BIBLIOGRÁFICAS}

1. Ministerio de Sanidad y Consumo. Guía de práctica clínica sobre cuidados paliativos. Madrid: 2007.

2. Bayés R. Psicología del sufrimiento y de la muerte. Barcelona: Ediciones Martínez Roca; 2001.

3. Simón-Lorda P, Barrio-Cantalejo IM, Alarcos-Martínez FJ, Barbero-Gutiérrez J, Couceiro A, Hernando-Robles P. Ética y muerte digna: propuesta de consenso sobre un uso correcto de las palabras. Rev Calid Asist 2008;23:271-85. Doi: 10.1016/ S1134-282X(08)75035-8.

4. Barbero J, Díaz L. Diez cuestiones inquietantes en cuidados paliativos. An Sist Sanit Navar 2007;30(Supl. 3):71-89.

5. Barrreto P, Bayés R. El psicólogo ante el enfermo en situación terminal. Anales de Psicología 1990;6:169-80.

6. Ahmedzai $\mathrm{SH}$, Costa A, Blengini $\mathrm{C}$, Bosch A, Sanz-Ortíz J, Ventafridda V, et al. A new international framework for palliative care. Eur J Cancer 2004;40:2192-200. Doi: 10.1016/j.ejca.2004.06.009

7. Ministerio de Sanidad PSel. Estrategia en Cuidados Paliativos del Sistema Nacional de Salud. Actualización 2010-2014. Madrid: Ministerio de Sanidad, Política Social e Igualdad, 2011.

8. Lacasta MA. Los psicólogos en los equipos de cuidados paliativos en el plan integral de la comunidad de Madrid. Psicooncología 2008;5:171-7.

9. Lacasta MA, Rocafort Gil J, Blanco Toro L, Limonero García JT, Gómez Batiste X. Intervención psicológica en Cuidados Paliativos. Análisis de los servicios prestados en España. Med Paliat 2008;15:39-44.

10. Barbero J. Psicólogos en Cuidados Paliativos: La sinrazón de un olvido. Psicooncología 2008;5:179-91.

11. Bayés R. Medicina Paliativa: psicología y Cuidados Paliativos. Med Pal 2005;12:137-8.

12. $\mathrm{Yi} \mathrm{P}$, et al. Grief support provided to caregivers of palliative care patients in Spain. Palliat Med 2006;20:521-31. Doi: 10.1191/0269216306pm1165oa

13. Haley W, Larson DG, Kasl-Godley J, Neimeyer RA, Kwilosz DM. Roles for psychologist in end-of-life care: Emerging models of practice. Profess Psych Research Pract 2003;34:626-33. Doi: 10.1037/07357028.34.6.626

14. Payne S, Haines R. The contribution of psychologist to specialist palliative care. Int J Palliat Nurs 2002;8:401-6.

15. Werth Jr JL, Lewis MM, Richmond JM. Psychologists' involvement with terminally ill individuals who are making end-of-life decisions. J For Psych Pract 2009;9:8291. Doi:10.1080/15228930802427130

16. Werth Jr JL, Gordon JR, Johnson Jr R. Psychosocial issues near the end of life. Aging Ment Health 2002;6:402-12. Doi:10.1080/1360786021000007027

17. Augé C. Psychologue en soins palliatifs: de la place du Sujet à l'hôpital. INFOKara 2001;63:15-7. 
18. Laval G, Villard ML, Comandini F. Qu'attend-on d'un psychologue en equipe mobile de soins palliatifs? Son rôle et ses missions. Presse Médicale 2003;15:67782.

19. Serra M. Psicólogo en Paliativos. Nuevo Rol Med Pal 2003;10:215-9.

20. Jünger $S$, Payne $S$, Costantini $A$, Kalus $C$, Werth Jr JL. The EAPC Task Force on Education for Psychologist in Palliative Care. Eur J Palliat Care 2010;17:84-7.

21. Payne S, Haines R. Doing our bit to ease the pain. Psychologist 2002;15:564-7.

22. Ortega Ruiz C, López Ríos F, Gómez Martín S. Intervención psicológica sobre el desgaste profesional de los profesionales sanitarios de la Unidad de Cuidados Paliativos del Hospital Universitario Gregorio Marañón. Med Pal 2008;15:93-7.

23. Lacasta MA, Sanchez Sobrino M, Nuñez Olarte JM, Gándara A, Gómez Batiste X. El psicólogo en los equipos de Cuidados Paliativos. Med Pal 2006;13:4.

24. Barrreto P, Soler MC. Psicología y fin de vida. Psicooncología 2003;0(1):135-46.

25. Fernández Fernández ME, Aguirán Clemente P, Amurrio López de Gastian LM, Limonero García JT, Martínez García M, Urbano Salgado S. Estándares de formación psicológica en Cuidados Paliativos. Med Pal 2004;11:174-9.

26. Fernández Fernández ME. Formación en Cuidados Paliativos por y para el psicólogo. Med Pal 2003;10:39-46.

27. DeAngelis T. More psychologist needed in end-of-life care. Monitor on Psychology 2002;33:3.

28. Twillman RK. The role of psychologists in palliative care. J Pharmaceut Care Pain Symptom Contr 2001;9:79-83.

29. Fernández-Alcántara M, García-Caro MP, Pérez-Marfil N, Cruz-Quintana F. Experiencias y obstáculos de los psicólogos en el acompañamiento de los procesos de fin de vida. Anal Psicol 2013;29:1-8. Doi: 10.6018/analesps.29.1.139121
30. Nydegger R. Psychologist and Hospice: where we are and where we can be. Profess Psych Research Pract 2008;39:459-63.

31. Alexander P. An investigation of inpatient referrals to a clinical psychologist in a hospice. Eur J Cancer Care (Engl) 2004;13:3644.

32. Romero C, Álvarez M, Bayés R, Schröder M. ¿Cuándo se pide la intervención del psicólogo en una unidad de Cuidados Paliativos? Med Pal 2001;8:170-2.

33. Lloyd-Williams M, Road G, Friedman T. A survey of psychosocial service provision within hospices. Palliat Med 1999;13:4312. Doi: 10.1191/026921699672181597

34. Lacasta MA. Módulo de análisis de la situación actual de los psicólogos de Cuidados Paliativos en España. Med Pal 2001;8:198-205.

35. Doucet CRP. L'acte clinique en soins palliatifs au regard de I'intime et du seuil de la pudeur. Med Pall (Paris) 2009;9:137-41. Doi: 10.1016/j.medpal.2009.10.003

36. Bonnaud-Antignac A, Ferreol M. Rôle du psychologue face à la violence de la fin de vie en soins palliatifs. L'evolution psychiatrique 2008;73:509-16.

37. Cavro É. Que deviennent le rôle et la cadre du psychologue dans les situations où l'information ne cirule pas? Med Pall (Paris) 2008;7:207-10. Doi: 10.1016/j. medpal.2008.05.006

38. Le Jamtel C. Polyvalence et ambivalence du psychologue en soins palliatifs. Med Pall (Paris) 2008;7:203-6. Doi: 10.1016/ S1636-6522(07)79708-8

39. Leboul D. Être psychologue en soins palliatifs: maintenir une pensée cohérente malgré la polyvalence del fonctions. Med Pall (Paris) 2004;3:307-10. Doi: 10.1016/ S1636-6522(04)97899-3

40. Bioy A. Culture et dépendance du psychologue en soins palliatifs. Med Pall (Paris) 2008;7:199-202. Doi: 10.1016/j.medpal.2008.05.007

41. Brocq H. Le psychologue et la relation d'aide en clinique palliative: un 
complément indispensable à la prise en charge médico-chirurgicale. Med Pall (Paris) 2005;4:4-9. Doi: 10.1016/S16366522(05)81553-3

42. Doucet C. L'intevention du psychologue clinicien en soins palliatifs: une pratique orientee par la psychanalyse. Revue Francophone de Psycho-Oncologie 2003;2:914.

43. Michaud A, Triol I. Le soin palliatif, la mort et le psychologue. Med Pall (Paris) 2004;3:257-61. Doi: 10.1016/S16366522(04)97885-3

44. Stadelmaier N, Lombard I, Ferreol M. Les transmissions écrites des psychologues en soins palliatifs: quels enjeux ? Med Pall (Paris). 2011;10:130-6. Doi: 10.1016/j. medpal.2010.07.002

45. Martin-Primat E, Richard A. Soins palliatifs et Psychologie. Le vécu psychologique des médecins, infirmières et psychologues en EMSP. Enquête. Med Pall (Paris) 2008;7:917. Doi: 10.1016/j.medpal.2007.07.001

46. Ibánez-del Prado C, Díaz-Sánchez R, Díaz-Hernández E. ¿Qué es para usted un psicólogo? Responden enfermos y cuidadores principales. Med Paliat 2012;19:8794. Doi: 10.1016/j.medipa.2010.11.008

47. Duro-Martínez JC, De Miguel C, Morillo E, Noguera A, Núñez-Portela B, RuizLópez D. Discurso de los profesionales de cuidados paliativos de la comunidad de madrid sobre la atención psicológica. Psicooncología 2012;9:467-81. Doi: 10.5209/rev_PSIC.2013.v9.n2-3.40918

48. Barreto $\mathrm{P}$, de la Torre $\mathrm{O}$, Pérez-Marin $\mathrm{M}$. Detección de duelo complicado. Psicooncología 2012;9:355-68. Doi: 10.5209/ rev_PSIC.2013.v9.n2-3.40902

49. Marwit SJ. Professional psychology's role in hospice care. Profess Psych Research Pract 1997;28:457-63.

50. Ramsay N. Sitting Close to Death: Observation on a Palliative Care Unit. Group Analysis 1995;28:335-65. Doi: 10.1177/0533316495283011

51. Ramsay N. Referral to a liaison psychiatrist from a palliative care unit. Palliat Med 1992;6:54-60. Doi: 10.1177/026921639200600109 